\title{
Understanding the genetic basis of potato development using a multi-trait QTL analysis
}

\author{
P. X. Hurtado-Lopez • B. B. Tessema $\cdot$ S. K. Schnabel • \\ C. Maliepaard - C. G. Van der Linden - P. H. C. Eilers • \\ J. Jansen · F. A. van Eeuwijk • R. G. F. Visser
}

Received: 10 April 2014/Accepted: 18 March 2015/Published online: 1 April 2015

(C) The Author(s) 2015. This article is published with open access at Springerlink.com

\begin{abstract}
Understanding the genetic basis of plant development in potato requires a proper characterization of plant morphology over time. Parameters related to different aging stages can be used to describe the developmental processes. It is attractive to map these traits simultaneously in a QTL analysis; because the power to detect a QTL will often be improved and it will be easier to identify pleiotropic QTLs. We included complex, agronomic traits together with plant development parameters in a multi-trait QTL analysis. First, the results of our analysis led to coherent insight into the genetic architecture of complex traits in potato. Secondly, QTL for parameters related to plant development were identified. Thirdly, pleiotropic regions for various types of traits were identified. Emergence, number of main stems,
\end{abstract}

P. X. Hurtado-Lopez · B. B. Tessema .

C. Maliepaard - C. G. Van der Linden - R. G. F. Visser Wageningen UR Plant Breeding, Wageningen University \& Research Centre, Wageningen, The Netherlands e-mail: p.hurtado.lopez@ rijkzwaan.nl

B. B. Tessema

e-mail: biructawit.tessema@wur.nl

C. Maliepaard

e-mail: chris.maliepaard@wur.nl

C. G. Van der Linden

e-mail: gerard.vanderlinden@wur.nl

R. G. F. Visser

e-mail: richard.visser@wur.nl number of tubers and yield were explained by $9,5,4$ and 6 QTL, respectively. These traits were measured once during the growing season. The genetic control of flowering, senescence and plant height, which were measured at regular time intervals, was explained by 9 , 10 and 12 QTL, respectively. Genetic relationships between aboveground and belowground traits in potato were observed in 14 pleiotropic QTL. Some of our results suggest the presence of QTL-byEnvironment interactions. Therefore, additional studies comparing development under different photoperiods are required to investigate the plasticity of the crop.

Keywords Development - Multi-trait analysis · Plant development $\cdot$ Pleiotropy $\cdot$ Potato $\cdot$ Senescence

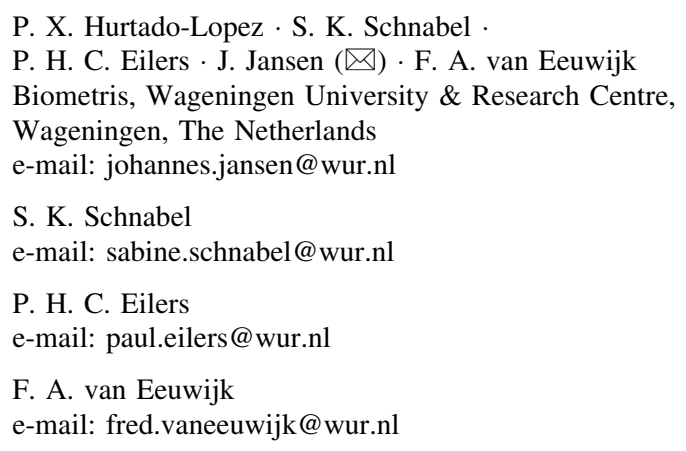




\section{Introduction}

The development of plants is a complex, dynamic process controlled by networks of genes as well as environmental factors. As a consequence, QTL analysis of traits related to plant development requires the use of advanced statistical-genetic models and methods (Atchley 1984; Wolf et al. 2001). Conventional QTL mapping strategies neglect the fact that traits related to plant development are changing in time. For example, in potato plant height and tuber size change in time, and their development is influenced by changing environmental factors during the growth season. Therefore, such traits should be represented by functions of time and/or variables describing the major changes in environmental factors over time. This requires an approach that is able to detect genetic effects related to plant development.

In Arabidopsis, molecular markers have been associated with phenotypes observed at different development stages and the differences between these stages have been compared (Mauricio 2005). In the same model plant, simulated time series data have been used to infer growth curves in order to study the quantitative nature of plant development (Mundermann et al. 2005). A more general strategy to study the genetic architecture of complex, dynamic traits, socalled functional mapping, has been proposed to integrate the development of traits in time into QTL mapping (Lin and $\mathrm{Wu}$ 2006; Wu and Lin 2006;

P. X. Hurtado-Lopez

C.T. de Wit Graduate School for Production Ecology and Resource Conservation (PE\&RC), Wageningen

University, Wageningen, The Netherlands

Present Address:

P. X. Hurtado-Lopez

RijkZwaan R\&D, Fijnaart, The Netherlands

B. B. Tessema

The Graduate School Experimental Plant Sciences (EPS), Wageningen University, Wageningen, The Netherlands

S. K. Schnabel · J. Jansen · F. A. van Eeuwijk ·

R. G. F. Visser

Centre for BioSystems Genomics, Wageningen,

The Netherlands

P. H. C. Eilers

Erasmus University Medical Center, Rotterdam, The Netherlands
Wu et al. 2003). Dissecting the genetic basis of plant development requires an accurate description of developmental morphology. Such descriptions are often lacking and conclusions are drawn based on observations of fully grown plants (Kellogg 2004). This means that comparisons between developmental phases are often superficial. Therefore, a proper characterization of development over time is needed to describe each part of the process.

In potato, previous studies have incorporated well characterised time series data into growth models and QTL analysis. This approach allowed a genetic description of senescence in terms of parameters related to different aging stages (Hurtado et al. 2012; Malosetti et al. 2006). To our knowledge, studies embedding plant development in potato into a simultaneous QTL analysis with complex, agronomic traits have not been reported. Therefore, the genetic control of plant development is still poorly understood.

Although many QTL studies considered multiple traits, usually those traits were analysed separately. An integrated analysis combining traits related to developmental processes simultaneously is required to get a better understanding of the genetic and environmental forces driving plant development. QTL analysis combining data from multiple traits related to plant development will not only increase the power of QTL detection, it will also improve the understanding of the genetic control of developmental processes. As a consequence, a multi-trait QTL analysis of a single population allows the detection of closely linked chromosomal regions affecting several traits simultaneously (Jiang and Zeng 1995). Although different methodologies have been proposed not only to map multiple trait simultaneously (Jiang and Zeng 1995; Knott and Haley 2000; Malosetti et al. 2008) but also to differentiate between close linkage and pleiotropy of coincident QTL (Jiang and Zeng 1995; Knott and Haley 2000; Lebreton et al. 1998; Liu et al. 2007), the identification of pleiotropic genes requires additional genomic information such as high density linkage maps and genome sequence information.

A first attempt to estimate the optimal set of consensus QTL for several traits simultaneously in potato was done through a QTL meta-analysis (Danan et al. 2011). It permitted the co-localization of late blight resistance and plant maturity traits by projecting individual QTL onto a consensus map. However, there are no reports of such integrative analysis for 
developmental traits in potato. So far, data on traits related to plant development in potato have not been integrated in a single study in order to get insight into the genetic architecture of crop development and the presence of putative pleiotropic QTL related to plant development.

The aim of this study was to identify the genetic basis of plant developmental processes in potato by means of a multi-trait QTL analysis combining several traits describing plant development in time. A total of 23 traits related to plant development and agronomic value were incorporated in the multi-trait QTL analysis. For this purpose, a diploid potato mapping population was evaluated under field conditions. Plant height, flowering and senescence were assessed on a weekly basis. The agronomic traits yield, number of main stems and number of tubers were measured at harvest. We were interested in the presence and genetic positions of putative pleiotropic regions associated with plant development and traits of agronomic value. Fourteen pleiotropic QTL were detected in our study, providing insights into the genetic architecture of developmental processes and the genetic relationship between above and below ground traits in potato. The anchoring of putative pleiotropic QTL to the annotated potato genome sequence (The Potato Genome Sequencing Consortium 2011) will provide target genes for marker assisted breeding and candidate gene approaches.

\section{Materials and methods}

Plant materials

Potato development was assessed in the diploid backcross population, hereafter referred to as $\mathrm{C} \times \mathrm{E}$. It was obtained from a cross between clone C (USW5337.3 (Hanneman and Peloquin 1967); a hybrid between Solanum phureja (PI225696) and a dihaploid S. tuberosum (US-W42)) and clone E (a hybrid between $\mathrm{VH}^{3} 4211$ (a $S$. vernei-S. tuberosum backcross) and clone $\mathrm{C}$ ). $\mathrm{C} \times \mathrm{E}$ was developed for research purposes (Jacobs et al. 1995) based on the genetic background of the parents. It is known for its segregation of agronomic and quality traits (CelisGamboa 2002; Kloosterman et al. 2010). S.tuberosum and $S$. phureja have different day length requirements for tuberization making $\mathrm{C} \times \mathrm{E}$ suitable for the study of developmental processes influenced by photoperiod and other environmental conditions. In total, 190 genotypes were used in the experiment: parents $\mathrm{C}$ and E, 169 genotypes of $\mathrm{C} \times \mathrm{E}$, a selected group of nine European cultivars ('Astarte', 'Bintje', 'Gloria', 'Granola', 'Karnico', 'Mondial', 'Première', 'Saturna' and 'Desiree') and 10 Ethiopian cultivars ('Awash', 'Belete', 'Bulle', 'Gera', 'Gorebella', 'Guassa', 'Gudene', 'Jalene', 'Shenkolla' and 'Zengena').

\section{Experimental setup}

The $\mathrm{C} \times \mathrm{E}$ population was planted in a light clay soil under rain fed conditions on July16 2010 at Holetta Agricultural Research Center, Ethiopia $\left(9.07^{\prime} \mathrm{N}\right.$, $38.03^{\prime} \mathrm{E}$ in West Ethiopia at an altitude of $2400 \mathrm{~m}$ ). Planting was done by hand, with a spacing of $75 \mathrm{~cm}$ between rows and $30 \mathrm{~cm}$ within rows. Fertilizer (165 kg UREA and $196 \mathrm{~kg}$ diammonium phosphate per hectare) was applied during planting and a fungicide (RidomilGold) was sprayed against late blight. Ridging was carried out three times throughout the experiment and weeding was done by hand whenever necessary. The experiment was laid out in a randomized complete block design with three blocks, laid against the slope of the field. In each block, the two parents, the $\mathrm{C} \times \mathrm{E}$ genotypes and the European and Ethiopian varieties were randomized over 190 plots, with 4 plants per plot. The observation period of the developmental traits was 5 months (between July and December 2010) and meteorological data were obtained during this period from the meteorological service present at the research station. The air temperature was recorded daily, every $3 \mathrm{~h}$, day and night. Over the whole observation period, the temperature fluctuated between 4 and $23{ }^{\circ} \mathrm{C}$ between $6 \mathrm{am}$ and $6 \mathrm{pm}$ and during the night between 2 and $20{ }^{\circ} \mathrm{C}$. During the experiment the day length was $12 \mathrm{~h}$.

\section{Agronomic traits}

During the growing period, for each plant the development was assessed by measuring aboveground and belowground traits. Aboveground, the date of emergence and the number of main stems were assessed once, while plant height, flowering and senescence were measured over time at regular intervals. Belowground, number of tubers and total tuber weight were assessed after the final harvest. 
The evaluation of flowering and senescence was done using a scale from 0 to 7 and 1 to 7 respectively, as described in (Celis-Gamboa et al. 2003). Flowering was recorded 17 times with intervals of 2-6 days at 38, 40, 42, 45, 47, 49, 52, 54, 56, 59, 61, 63, 66, 68, 70, 74, $80,83,87,89$ and 95 days after planting (DAP). Senescence was assessed 16 times with intervals of 3-7 days at $(80,83,87,91,95,99,103,107,111,115$, 119, 123, 129 and 136 DAP.

Plant height was measured using the longest stem of each plant as the distance from ground level to main apex. The assessment was done at nine occasions with intervals of 6 days $(26,32,38,44,50,56,62,68$ and 74 DAP). All plots were harvested at 138 DAP and the tubers of each plant were counted and weighed.

\section{Conversion of days after planting into thermal days}

Crop development is mainly affected by temperature and can be modified by other factors such as photoperiod (Hodges 1990). Previous potato studies have shown that warm conditions lead to an acceleration of vegetative and reproductive development (flowers, berries) (Benoit et al. 1986; Haun 1975; Struik and Ewing 1995), whereas cooler conditions facilitate tuber growth (Marinus and Bodlaender 1975). The effect of temperature on crop development rate is often described by using a thermal-time concept. Thus, various non-linear models have been developed to describe the temperature response of developmental processes in plants (Gao et al. 1992; Johnson and Thornley 1985; Yin et al. 1995). In our study, fluctuations in temperature under field conditions were accounted for by estimating the daily contribution of temperature to plant development. Calendar days after planting were transformed into thermal days after planting (TAP) using the non-linear temperature effect beta-function described by Yin et al. (1995). Day length was incorporated into this function as a constant (Masle et al. 1989). This was done to anticipate on a later comparison of the performance of the $\mathrm{CxE}$ population under different day length conditions. The non-linear relationship between temperature, photoperiod and rate of growth is described by

$g\left(T_{i}\right)=\left[\left(\frac{T_{c}-T_{i}}{T_{c}-T_{o}}\right)\left(\frac{T_{i}-T_{b}}{T_{o}-T_{b}}\right)^{\frac{T_{o}-T_{b}}{T_{c}-T_{o}}}\right]^{c_{t}} l_{i}$ in which the three cardinal temperatures for phenological development of potato (base: $T_{b}$, optimal: $T_{o}$ and ceiling: $T_{c}$ ) and the temperature response curvature coefficient, $c_{t}$, have been assigned the values $T_{b}=5.5{ }^{\circ} \mathrm{C}, T_{o}=23.4{ }^{\circ} \mathrm{C}, T_{c}=34.6{ }^{\circ} \mathrm{C}$ and $c_{t}=$ 1.7, respectively (Khan 2012; Khan et al. 2013). $T_{i}$ is the average daily air temperature and $l_{i}$ is the light period as a proportion of a day on day $i$ after planting. The new thermal unit is then the cumulative betathermal days after planting combining, temperature, time and photoperiod (photo-beta thermal time, PBTT). This scale was used as the $\mathrm{x}$-axis to analyse the time series data of plant height, flowering and senescence. PBTT will allow a better comparability of the traits across years and locations than normal time.

\section{Curve fitting and characterization of the curves}

Curve fitting of plant height, flowering and senescence was done using PBTT units on the $\mathrm{x}$-axis. For modelling flowering and senescence we used a methodology previously described to fit senescence data in potato (Hurtado et al. 2012). A smooth generalized linear model was used to estimate smooth curves for the development of flowering and senescence over time. The estimation was done using the $\mathrm{R}$ software environment (CoreTeam 2011). A different approach was used to model plant height. In contrast to flowering and senescence, plant height was measured as a continuous variable (in $\mathrm{cm}$ ). Up to twelve observations per genotype were available per time point. We pooled the 12 observations per genotype in each time point and fitted a curve to the relationship between plant height and time. A smooth expectile curve was well suited for this purpose and the expectiles were estimated using least asymmetrically weighted squares (Schnabel and Eilers 2009). They were combined with P-splines to provide a flexible functional form (Schnabel et al. 2012). This modelling procedure resulted in a smooth frontier curve to describe the development of plant height over time. For the calculations we used the package "expectreg" in R (Sobotka et al. 2012).

Parameters describing the development process were estimated by fitting the development curves to data. These parameters facilitated the study of development as a continuous process in time by breaking down the complex traits into components related to the 


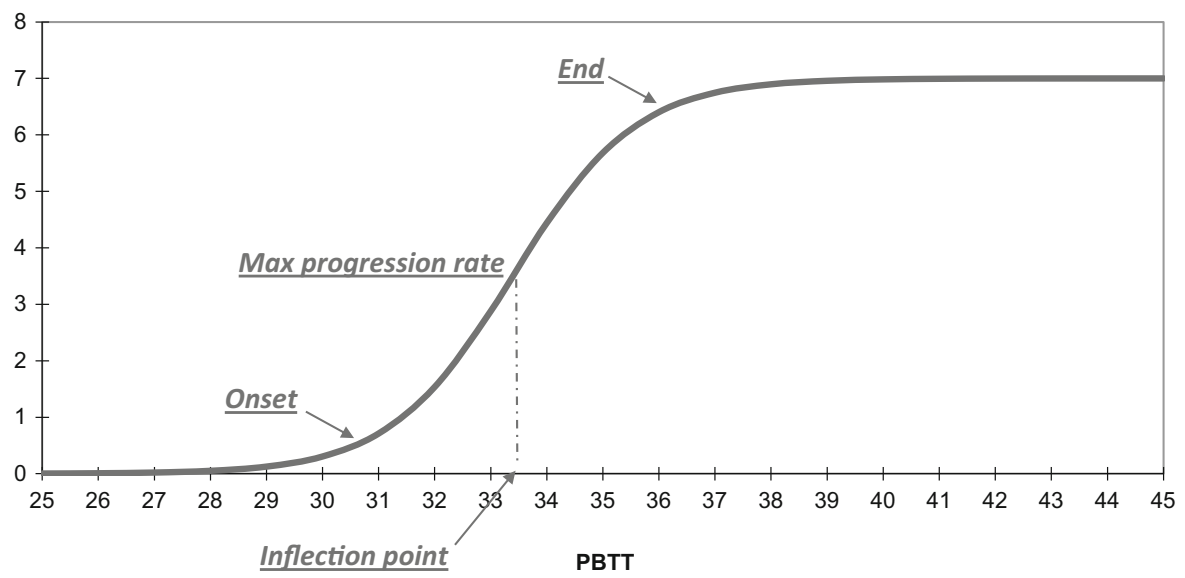

Fig. 1 Fitted curve for flowering development of a random genotype of the $\mathrm{C} \times \mathrm{E}$ population. It is used as example to show the parameters describing flowering, senescence and plant height. On the x-axis: photo-beta thermal time (PBTT), on the y-axis: flowering on a scale from 0 to 7

different developmental stages. The first and second derivative of the fitted curves have been used to characterise senescence processes under long day length conditions (Hurtado et al. 2012). The parameters used to characterise senescence were also used in our study to describe plant height, flowering and senescence under short photoperiod (Fig. 1). These parameters are onset of development, mean and maximum progression rates (average and maximum speed of the development process), inflection point or the turning point at which the process enters into the final phase, and end of development. We also considered additional traits describing growth and development, such as maximum and mean plant height, duration of flowering and maximum progression rate for onset of plant height (maximum speed of the process between emergence and the first observation of plant height). Note that the parameters have different units and their interpretation is different. For instance, small values of progression rate indicate slow flowering, senescence or plant height processes, mainly associated to late genotypes; while small values of inflection point, onset or end are related to early genotypes.

Genetic maps and molecular data

Single nucleotide polymorphism (SNP) markers scored in a core set of $\mathrm{C} \times \mathrm{E}$ (Anithakumari et al. 2010) were added to the maps of parents $C$ and $E$ as described in Hurtado et al. (2012). Together with the
SNP markers, AFLP, SSR and CAPS with expected segregation ratios $1: 1$ and $1: 1: 1: 1$, respectively, were used to construct more saturated maps of parent $\mathrm{C}$ and E (additional file 1). JoinMap 4 (van Ooijen 2009) was used to map 521 and 560 markers on the $\mathrm{C}$ and $\mathrm{E}$ maps, respectively, with 12 linkage groups (LG) for each parent as reported previously (Celis-Gamboa 2002).

Considering the differences in the recombination frequencies between the two parents (due to the fact that they originated from two different Solanum species), the $\mathrm{C}$ and $\mathrm{E}$ maps were not integrated. Markers segregating 1:1 and 1:1:1:1, were used in the QTL analysis; the latter ones were converted into two 1:1 types by separating the parental meioses in accordance with a pseudo-testcross analysis (Grattapaglia and Sederoff 1994).

\section{Multi-trait QTL analysis}

Two types of phenotypic traits were considered in our study (Table 1): growth and senescence curve parameters and agronomic plant characters measured on a single occasion during the growing season. For the agronomic traits, genotypic means were obtained from a linear model with blocks (three levels) and genotypes (169 levels). The curve parameters and the genotypic means for the agronomic traits were analysed together in a multi-trait QTL analysis (Alimi et al. 2013; Jiang and Zeng 1995; Stephens 2013). 
Table 1 Phenotypic traits included in the multi-trait QTL analysis, trait units and developmental processes

\begin{tabular}{llll}
\hline Trait type & Traits & Units & Developmental processes \\
\hline $\begin{array}{l}\text { Parameters derived from } \\
\text { fitted curves }\end{array}$ & Onset & Thermal days & Flowering, senescence, plant height \\
& Maximum progression rate & & Flowering, senescence, plant height \\
& Inflection point & Thermal days & Flowering, senescence, plant height \\
& End & Thermal days & Flowering, senescence, plant height \\
& Mean progression rate & & Flowering, senescence, plant height \\
Maximum progression rate in onset & & Plant height \\
once during the growing season & Duration of flowering & Days after planting & Flowering \\
& Maximum height & $\mathrm{cm}$ & Plant height \\
& Mean height & $\mathrm{cm}$ & Plant height \\
& Emergence & Days after planting & \\
& Number of main stems & Number & Number \\
& Total number of tubers & Kg & \\
& Yield & &
\end{tabular}

including 23 traits: five common traits for the three developmental processes (onset, maximum progression rate, inflection point, end and mean progression rate), one additional trait describing flowering (duration of flowering), three additional traits related to plant height (maximum progression of onset, maximum and mean height) and four agronomic traits (emergence, number of main stems, total number of tubers and yield). All the traits were standardized (subtracting the average and dividing by the standard deviation) to make traits with different scales and units comparable for the multi-trait analysis.

For the multi-trait QTL analysis, the C and E maps were combined in a single map with linkage groups $\mathrm{C} 1, \ldots, \mathrm{C} 12$ and $\mathrm{E} 1, \ldots, \mathrm{E} 12$. This allowed the use of markers of one parent as co-factors while searching for QTL in the other parent, thereby increasing the power to detect QTL. The QTL library of Genstat 15 (VSNi 2012) was used for the multi-trait QTL analysis by fitting the models as described by van Eeuwijk et al. (2010) and Alimi et al. (2013). The analysis started by fitting QTL models using simple interval mapping, SIM (Lander and Botstein 1989). The model that was fitted in SMI was; trait $=$ trait intercept + trait specific QTL + residual genotypic effect + error. The residual genetic effects followed a multivariate normal distribution with an unstructured variance-covariance matrix.

The significance of trait-specific QTL was tested by a Wald test (Verbeke and Molenberghs 2000). A multiple testing correction was based on a Bonferroni procedure where effective number of tests is estimated from the genotype by marker score matrix as described in $\mathrm{Li}$ and $\mathrm{Ji}$ (2005), with a genome-wide test level of 0.05. A trait-specific confidence interval for QTL location was calculated according to Darvasi and Soller (1995). We adapted this procedure to the multitrait context by choosing the shortest confidence interval among the individual traits following the original prescription to define the interval for all traits simultaneously (Alimi et al. 2013). We followed the strategy described by Boer et al. (2007) and Malosetti et al. (2013) to arrive at a final multi-QTL model; first a SIM scan was performed to identify a set of candidate QTL. The candidate QTL from the SIM scan were used as co-factors in a composite interval scan. After the composite interval scan, a backward elimination round was used to remove possibly redundant QTL. The percentage variance explained by a QTL was calculated as the square of the allelic substitution effect divided by the phenotypic variance based on trial means, multiplied by 100 (to obtain a percentage); this implicitly assumes a 1:1 segregation of the alleles at the QTL.

\section{Results}

Curve fitting and characteristics of the curves

Curves describing development over time were fitted to the data of the individuals of $\mathrm{C} \times \mathrm{E}$, parents $\mathrm{C}$ and $\mathrm{E}$, 


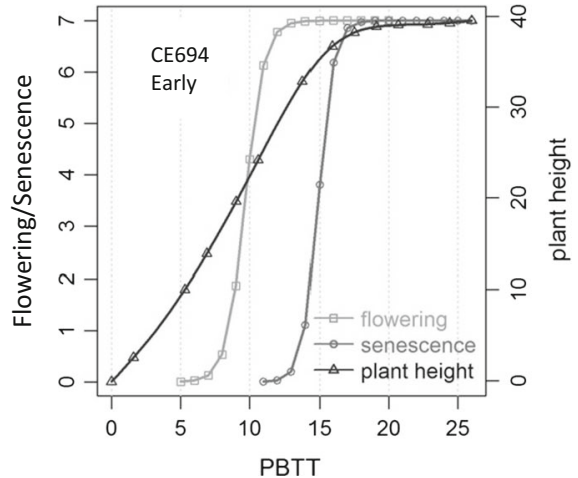

Fig. 2 Fitted curves for plant height, flowering and senescence of two genotypes representing early and late maturing groups. On the x-axis: PBTT (Photo-beta thermal time) units combining

and the control varieties. Differences in curve trajectories were observed between early and late genotypes for flowering, senescence and plant height (Fig. 2). The maturity type of $\mathrm{C} \times \mathrm{E}$ was previously assessed under field conditions (Celis-Gamboa 2002) and it was used as reference in the present study. Early genotypes completed their life cycle faster and a complete $\mathrm{S}$-shaped curve could be observed. Late genotypes showed slow progression of the developmental traits and some of them did not even complete the flowering and aging processes during the observation period. In that case, only the first part of the $\mathrm{S}$ shape could be observed.

In $\mathrm{C} \times \mathrm{E}$ a direct relationship was found between growth and maturity. Most of the late genotypes were tall and the early genotypes were short. However, the relationship between plant height and maturity did not hold for the Dutch cultivars (data not shown). For instance, Dutch varieties, irrespective of their maturity type, showed fast progression of senescence and all of them were shorter than the Ethiopian cultivars. This indicates that in these varieties maturation was accelerated whereas growth was restricted under short day conditions. In addition, flowering curves could not be fitted for the Dutch varieties due to the absence of flowering or flower abortion. Thus, the reduction in photoperiod affected the Dutch varieties dramatically; they are adapted to long day lengths. Suppressed flower development was also observed in previous potato studies in growth chambers where the irradiance was reduced (Clarke and Lombard 1939; Turner and Ewing 1988). In all $\mathrm{C} \times \mathrm{E}$ genotypes flowering and senescence curves presented parallel trajectories and they overlapped in early

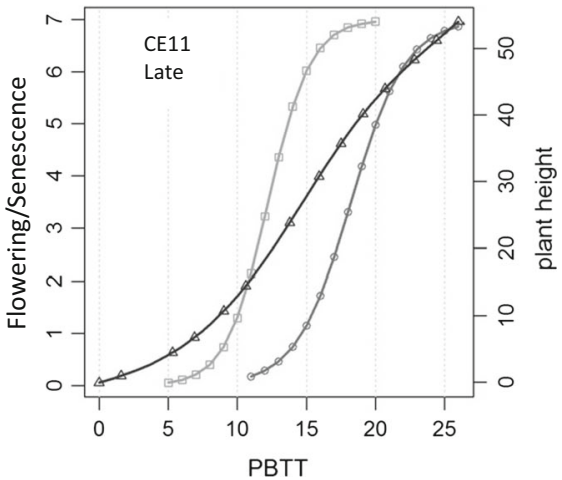

average daily air temperature and photoperiod. On the y-axis: flowering and senescence scales from 0 to 7 (left) and plant height in $\mathrm{cm}$ on a continuous scale (right)

genotypes at the final stage of both processes. Examples are given in Fig. 2.

Genetics of complex traits

The genetic architecture of complex developmental traits in potato was studied using the parameters derived from the fitted curves for flowering, senescence and plant height. Together with the agronomic traits they were included in a multi-trait QTL analysis and the QTL detected with the maternal and paternal maps could be observed in Fig. 2. Although our study mainly focused on the presence and positions of QTL (upper plot of Fig. 3) rather than on the allelic effects (lower plot), the QTL effects (positive: red; negative: blue) related to different values of the phenotypic traits, are also reported for the 23 traits on each QTL position. The size of QTL effects, indicated by the intensity of the colour (the darker the larger the effect), is also shown in Fig. 2 and the explained variance for each trait is provided in Table 2. Opposite effects within pleiotropic regions are expected for a QTL related to negatively correlated traits. For instance, progression of flowering is negatively correlated to end of flowering (Additional file 2) and QTL effect on C5 and E5 were observed for both traits. Plants with fast flowering development (high values for progression rate) are expected to have an early end of the flowering process (small values for end of flowering).

\section{Complex traits}

For each complex, agronomic trait multiple QTL were identified (Fig. 3). We checked the position of the 

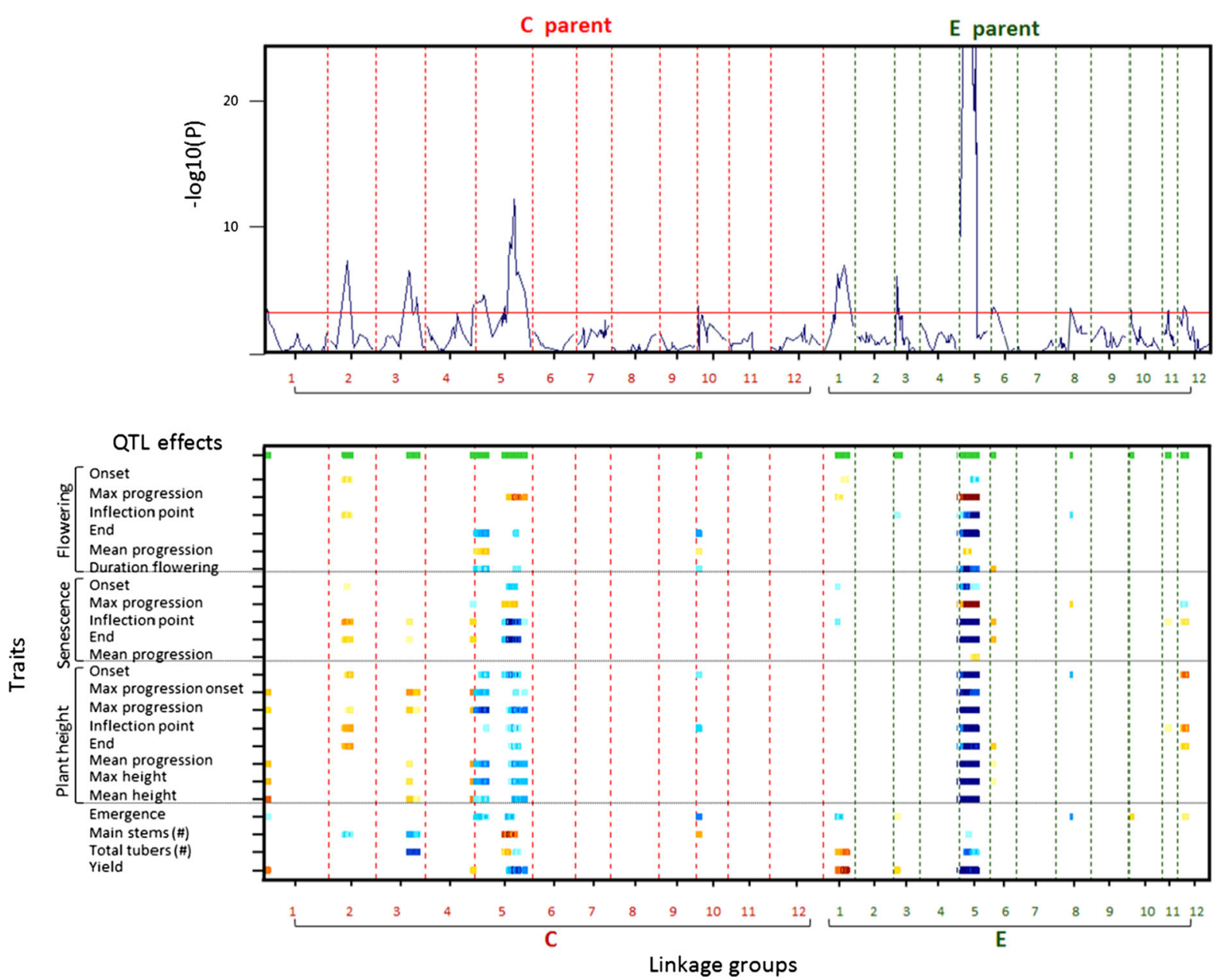

Fig. 3 Multi-trait QTL linkage analysis. The upper plot shows the significance of QTLs (- $\log 10$ scale for the associated probability value). The lower plot shows the positive (red) and negative (blue) allele substitution effects at positions where

QTL on the parental maps and the QTL detected on a particular linkage group were different from the QTL detected on the homologous linkage group in the other parent. Only one QTL was detected on C5 and E5 in the same genetic region. This was a major QTL associated with all developmental and agronomic traits (except emergence). In the E parent this QTL has a huge effect with values $-\log 10(\mathrm{p})$ going up to 50 ; for most traits, the explained variances for this QTL are very high going up to $60 \%$ for onset of senescence (Table 2). This finding is in agreement with previous reports indicating a major effect of a QTL in the same chromosomal region associated with plant maturity with pleiotropic effects on many developmental traits there was a significant QTL. The intensity of the colour is proportional to the QTL effect size (the darker the larger the effect). Only $-\log 10(\mathrm{p})$ values lower than 25 are presented in the figure

(Celis-Gamboa 2002; Hurtado et al. 2012; Kloosterman et al. 2013; Malosetti et al. 2006). According to our results there is no major contribution of this QTL to the agronomic traits as indicated by the low explained variances. Since our study focusses on new QTL (i.e. not the QTL on C5/E5 related to plant maturity) contributing to the understanding of the genetic architecture of complex traits, we have limited our discussion and main conclusions to those QTL.

\section{Flowering}

In our study the genetic control of flowering was driven by 9 QTL. The QTL on C2, E1, E3 and E8 were 
Euphytica (2015) 204:229-241

237

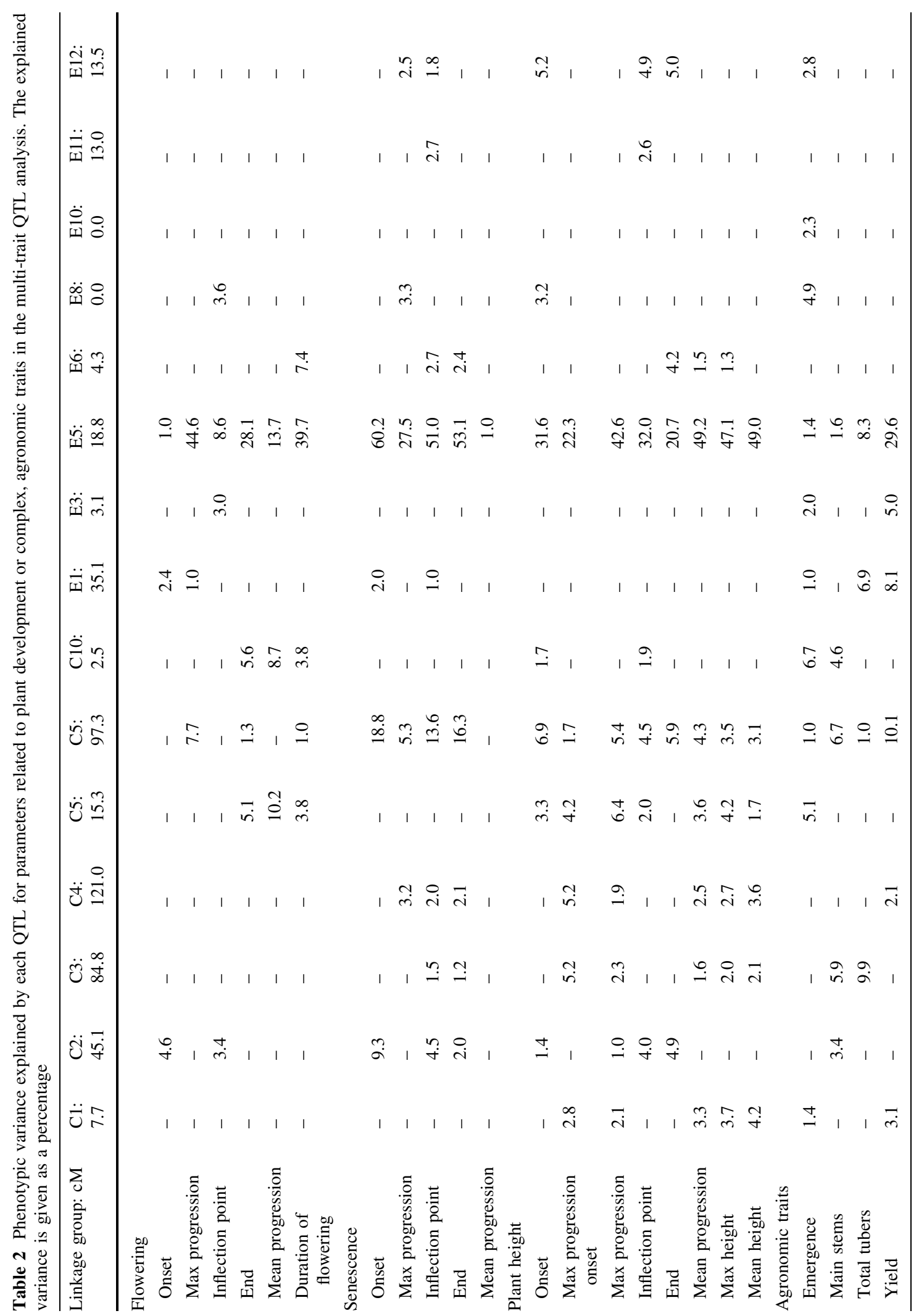

Springer 
associated with onset of flowering and other parameters of the flowering process (inflection point, maximum speed). The QTL on C10 and the first QTL on $\mathrm{C} 5$ with the total length of the flowering period and the end of flowering.

\section{Senescence}

in our study, ten QTL were found to be controlling the aging process. QTL on E1, E8 and E12 were related to onset of senescence and QTL on C3, C4 and E6 were associated with the end of senescence.

\section{Plant height}

We found 12 QTL related to plant height. QTL permanently expressed during the growing process were identified on $\mathrm{C} 2$, first half of C5, E5 and E12. QTL on C1, C3 and C4 were expressed between onset and half the growth process and they were also associated with the average and maximum plant height. The presence of common QTL for those traits could also be explained by the high phenotypic correlations between them (Additional file 2).

\section{Agronomic traits}

Emergence, number of main stems, total number of tubers and yield were explained by 9, 5, 4 and 6 QTL, respectively. These traits were measured once at the end of the growing season; therefore QTL related to the development of these traits could not be detected. Some QTL have been reported for yield on Chromosomes 1 and 6 in a tetraploid potato full-sib family (Bradshaw et al. 2008). In our study, QTL on C1 and E1 explained $11 \%$ of the phenotypic variance for yield suggesting the presence of a common genomic region on chromosome 1 in both parents for yield in potato.

Although there was an effect of chromosome 5 on the agronomic traits, it was smaller compared with the effect on developmental traits, except for yield (Table 2). These results suggest that plant maturity does not play a central role in the agronomic traits considered in our study.

Pleiotropic regions

The multi-trait QTL analysis combining developmental and agronomic traits not only increased the power of QTL detection, compared with single trait linkage analysis (Additional file 3), but it also helped us to detect pleiotropic regions controlling aboveground and belowground traits in potato.

Fourteen pleiotropic QTL associated with developmental and agronomic traits could be identified in our study. In parent $\mathrm{C}$, seven pleiotropic QTL were identified. For instance, the QTL on C2 was related with onset of plant height, flowering and senescence, progression of the three traits and number of main stems. The QTL on C3 was related to plant height, growth and number of tubers and number of main stems. In fact, previous studies have shown that tuber formation is reduced when the development of the haulm is accelerated (Maris 1964). A positive correlation between number of main stems and number of tubers has also been reported (Lemaga and Caesar 1990) but the g enetic control of these traits is not yet clear. Here, we are able to report for both traits a QTL on $\mathrm{C} 3$ explaining 6 and $10 \%$ of the phenotypic variance for number of main stems and total number of tubers, respectively. The QTL on C10 was associated with emergence, onset of growth, duration of flowering and number of main stems per plant. This QTL could facilitate the selection of high yielding varieties with fast growth and a short flowering period.

In the E parent, we detected one QTL on E10 associated with late emergence, seven pleiotropic QTL on E1, E3, E5, E6, E8, E11 and E12. For example, the QTL on E1 was associated with emergence, onset of senescence, number of tubers and yield, showing the highest explained variance for yield and number of tubers (8.1 and $6.9 \%$, respectively). The QTL on E8 was associated emergence, onset of growth and senescence. The QTL on E12 is affecting the same traits. The QTL on E6 and E11 affected senescence and plant height, but had no effect on the agronomic traits.

Further research will help to confirm the stability across environments of the pleiotropic regions associated with developmental traits found in our study and to investigate the presence of one or more genes in those regions.

\section{Discussion}

The curve fitting approaches followed in our study provided an effective characterization of the 
developmental processes that occur during the potato life cycle under short day length conditions. The parameters derived from the curves characterise different stages of the development of the above ground parts of the plant. Plant height, flowering and senescence are described by five parameters: onset, end, progression rate (average and maximum speed of the process) and inflection point (time point when half of the developmental process has been reached) These parameters can also be used to characterise other processes in which growth curves are fitted using discrete or continuous data collected as a time series. For some traits additional characteristics were taken into account, such as duration of flowering or maximum plant height and they were directly calculated from the data. We also considered an additional trait for plant height (progression rate between emergence and the first observation of plant height) that was estimated from the fitted curves. It shows that the methodology we used for curve fitting permits not only the characterization of the processes with the conventional parameters, but also the estimation of new characteristics according to the needs of the study.

Differences in trajectories were observed when comparing the fitted developmental curves according to earliness. In the case of flowering and senescence, early genotypes showed a complete S-shaped curve whereas late genotypes show slow progression and only the first part of the S-shape was observed in most of the genotypes. As already known, the genomic region on chromosome 5 controlling maturity has a pleiotropic effect on developmental traits (CelisGamboa 2002; Hurtado et al. 2012; Malosetti et al. 2006) and it can explain the curve's trajectories defined according to earliness. On the other hand, there was no clear relation between plant height and maturity as was also observed in a previous study (Maris 1964). Photoperiod played a role in both development and agronomic performance of the plants. This was specially observed in the Dutch varieties used as controls in the experiment. They were shorter compared with their height in the Netherlands and all of them showed fast senescence development indicating that under short day length, growth was restricted and maturation was accelerated. Another indication of the photoperiod effect on development was the flower abortion of the Dutch varieties. It is known that reduction in day length can suppress flower development (Turner and Ewing 1988).
To understand the genetic basis of the complex traits included in our study, developmental traits were treated as continuous and dynamic processes instead of looking at particular single moments of the life cycle. During the curve fitting all the time points were analysed together, a proper characterisation of different developmental stages was done and then the genetic factors underlying the processes were identified. A more efficient QTL analysis was performed using the estimated developmental parameters instead of searching for QTL per single time point. In addition, the number of QTL analyses was reduced. For instance, flowering was assessed in the field 17 times and we analysed only 6 parameters describing this trait. In the multi-trait QTL analysis presented here, all the parameters were analysed simultaneously and the presence of pleiotropic QTL was also investigated.

On the other hand, the combined use of parameters related to plant development and agronomic traits in a multi-trait QTL analysis provided coherent insight into (1) the genetic architecture of plant development and complex, agronomic traits in potato, (2) the presence of QTL for parameters related to plant development and (3) the genetic link between aboveground and belowground traits as discussed below.

For complex, agronomic traits, multiple QTL were identified explaining the genetic basis of these traits. Time-dependent QTL were detected for flowering, senescence and plant height. They showed a very low explained variance compared with the QTL expressed during the whole process (e.g. QTL related to mean progression rate). It has been reported that some QTL are expressed at early developmental stages and they are switched off after a particular age (Wu and Lin 2006). Time-dependent QTL have been observed in potato, controlling for instance onset and progression rate of senescence under long day length conditions (Hurtado et al. 2012).

We adapted the procedure of Darvasi and Soller (1995) to the multi-trait context by choosing the shortest confidence interval among the individual traits following the original prescription to define the interval for all traits simultaneously (Alimi et al. 2013). As one of the reviewers rightly mentioned, this may not be correct. Here we use it as a first approximation. We expect that our method is close to the true solution if for the trait concerned both the multi-trait analysis and the single-trait analysis put the QTL on the same position on the linkage map. 
Furthermore, our approximation will even be closer to the true solution if the sizes of the QTL effects in the single-trait analyses and the multi-trait analysis are approximately identical. For a general solution, however, which should also involve the covariance structure of the traits, more research is needed.

Further research will help (1) to confirm the stability of the pleiotropic regions found in our study across environments, (2) to check the consistency of the allele effects, which can vary according to the environmental setup where they are expressed (Clark 2000) and (3) to investigate the presence of genes in regions where evidence of QTL exists. Some of our results suggest the presence of QTL $\times$ Environment interactions; additional studies comparing development under different photoperiods are required to take full advantage of the plasticity of the crop. Multi-environment experiments will allow us to better quantify the effect of the different photoperiod on traits, such as the ones presented in this study. The paper provides a detailed description of powerful, statistical-genetic methods that may also be useful to other crop species. It provides results on potato genetics that will further enhance potato breeding.

Acknowledgments Part of this project was (co)financed by the Centre for BioSystems Genomics (CBSG) which is part of the Netherlands Genomics Initiative/Netherlands Organisation for Scientific Research. We are very grateful to the reviewers who raised questions which helped to improve the manuscript.

Conflict of interest The authors declare that they have no conflicts of interest.

Open Access This article is distributed under the terms of the Creative Commons Attribution License which permits any use, distribution, and reproduction in any medium, provided the original author(s) and the source are credited.

\section{References}

Alimi NA, Bink MCAM, Dieleman JA, Magán JJ, Wubs AM, Palloix A, van Eeuwijk FA (2013) Multi-trait and multienvironment QTL analyses of yield and a set of physiological traits in pepper. Theor Appl Genet 126:2597-2625

Anithakumari AM, Tang J, van Eck HJ, Visser RG, Leunissen JA, Vosman B, van der Linden CG (2010) A pipeline for high throughput detection and mapping of SNPs from EST databases. Mol Breed 26:65-75

Atchley WR (1984) Ontogeny, timing of development, and genetic variance-covariance structure. Am Nat 123:519-540
Benoit GR, Grant WJ, Devine OJ (1986) Potato top growth as influenced by day-night temperature differences 1 . Agron J 78:264-269

Boer MP, Wright D, Feng L, Podlich DW, Luo L, Cooper M, van Eeuwijk FA (2007) A mixed-model quantitative trait loci (QTL) analysis for multiple-environment trial data using environmental covariables for QTL-by-environment interactions, with an example in maize. Genetics 177:1801-1813

Bradshaw JE, Hackett CA, Pande B, Waugh R, Bryan GJ (2008) QTL mapping of yield, agronomic and quality traits in tetraploid potato (Solanum tuberosum subsp. tuberosum). Theor Appl Genet 116:193-211

Celis-Gamboa C (2002) The life cycle of the potato (Solanum tuberosum L.): from crop physiology to genetics. Plant Breeding. Wageningen University, Plant breeding department

Celis-Gamboa C, Struik PC, Jacobsen E, Visser RGF (2003) Temporal dynamics of tuber formation and related processes in a crossing population of potato (Solanum tuberosum). Ann Appl Biol 143:175-186

Clark M (2000) Comparative Genomics, 1st edn. Springer, Berlin

Clarke AE, Lombard PM (1939) Relation of length of day to flower and seed production in potato varieties. Am Potato J 16:236-244

CoreTeam RD (2011) R: a language and environment for statistical computing. R Foundation for Statistical Computing, Vienna

Danan S, Veyrieras JB, Lefebvre V (2011) Construction of a potato consensus map and QTL meta-analysis offer new insights into the genetic architecture of late blight resistance and plant maturity traits. BMC Plant Biol 11:16

Darvasi A, Soller M (1995) Advanced intercross lines, an experimental population for fine genetic mapping. Genetics 141:1199-1207

Gao L, Jin Z, Huang Y, Zhang L (1992) Rice clock model-a computer model to simulate rice development. Agric Forest Meteorol 60:1-16

Grattapaglia D, Sederoff R (1994) Genetic linkage maps of Eucalyptus grandis and Eucalyptus urophylla using a pseudo-testcross: mapping strategy and RAPD markers. Genetics 137:1121-1137

Hanneman R, Peloquin S (1967) Crossability of 24-chromosome potato hybrids with 48 -chromosome cultivars. Potato Res 10:62-73

Haun JR (1975) Potato growth-environment relationships. Agric Meteorol 15:325-332

Hodges T (1990) Temperature and water stress effects on phenology. Predicting Crop Phenology. Taylor \& Francis, Boca Raton, FL, pp 7-13

Hurtado PX, Schnabel SK, Zaban A, Vetelainen M, Virtanen E, Eilers PHC, van Eeuwijk FA, Visser RGF, Maliepaard C (2012) Dynamics of senescence-related QTLs in potato. Euphytica 183:289-302

Jacobs JME, Van Eck HJ, Arens P, Verkerkbakker B, Hekkert BTL, Bastiaanssen HJM, Elkharbotly A, Pereira A, Jacobsen E, Stiekema WJ (1995) A genetic map of potato (Solanum tuberosum) integrating molecular markers, including transposons, and classical markers. Theor Appl Genet 91:289-300

Jiang C, Zeng ZB (1995) Multiple trait analysis of genetic mapping for quantitative trait loci. Genetics 140:1111-1127 
Johnson IR, Thornley JHM (1985) Temperature-dependence of plant and crop processes. Ann Bot 55:1-24

Kellogg EA (2004) Evolution of developmental traits. Curr Opin Plant Biol 7:92-98

Khan MS (2012) Assessing genetic variation in growth and development of potato. Centre for crop systems analysis. Wageningen University, Wageningen, p 245

Khan M, van Eck H, Struik P (2013) Model-based evaluation of maturity type of potato using a diverse set of standard cultivars and a segregating diploid population. Potato Res 56:127-146

Kloosterman B, Oortwijn M, Uitdewilligen J, America T, de Vos R, Visser RGF, Bachem CWB (2010) From QTL to candidate gene: genetical genomics of simple and complex traits in potato using a pooling strategy. BMC Genom $11: 158$

Kloosterman B, Abelenda JA, Gomez MdMC, Oortwijn M, de Boer JM, Kowitwanich K, Horvath BM, van Eck HJ, Smaczniak C, Prat S, Visser RGF, Bachem CWB (2013) Naturally occurring allele diversity allows potato cultivation in northern latitudes. Nature 495:246-250

Knott SA, Haley CS (2000) Multitrait least squares for quantitative trait loci detection. Genetics 156:899-911

Lander ES, Botstein D (1989) Mapping Mendelian factors underlying quantitative traits using RFLP linkage maps. Genetics 121:185-199

Lebreton CH, Visscher PM, Haley CS, Semikhodskii A, Quarrie SA (1998) A nonparametric bootstrap method for testing close linkage versus pleiotrophy of coincident quantitative trait loci. Genetics 150:931-943

Lemaga B, Caesar K (1990) Relationships between numbers of main stems and yield components of potato (Solanum tuberosum L. cv Erntestolz) as influenced by different daylengths. Potato Res 33:257-267

Li J, Ji L (2005) Adjusting multiple testing in multilocus analyses using the eigenvalues of a correlation matrix. Heredity 95:221-227

Lin M, Wu R (2006) A joint model for nonparametric functional mapping of longitudinal trajectory and time-to-event. BMC Bioinform 7:138

Liu JF, Liu YJ, Liu XG, Deng HW (2007) Bayesian mapping of quantitative trait loci for multiple complex traits with the use of variance components. Am J Human Genet 81:304-320

Malosetti M, Visser RGF, Celis-Gamboa C, van Eeuwijk FA (2006) QTL methodology for response curves on the basis of non-linear mixed models, with an illustration to senescence in potato. Theor Appl Genet 113:288-300

Malosetti M, Ribaut JM, Vargas M, Crossa J, van Eeuwijk FA (2008) A multi-trait multi-environment QTL mixed model with an application to drought and nitrogen stress trials in maize (Zea mays L.). Euphytica 161:241-257

Malosetti M, Ribaut JM, van Eeuwijk FA (2013) The statistical analysis of multi-environment data: modeling genotypeby-environment interaction and its genetic basis. Front Physiol 4:44
Marinus J, Bodlaender KBA (1975) Response of some potato varieties to temperature. Potato Res 18:189-204

Maris B (1964) Studies concerning the relationship between plant height of potatoes in the seedling year and maturity in the clonal generations. Euphytica 13:130

Masle J, Doussinault G, Farquhar GD, Sun B (1989) Foliar stage in wheat correlates better to photothermal time than to thermal time. Plant, Cell Environ 12:235-247

Mauricio R (2005) Ontogenetics of QTL: the genetic architecture of trichome density over time in Arabidopsis thaliana. Genetica 123:75-85

Mundermann L, Erasmus Y, Lane B, Coen E, Prusinkiewicz P (2005) Quantitative modeling of Arabidopsis development. Plant Physiol 139:960-968

Schnabel SK, Eilers PHC (2009) Optimal expectile smoothing. Comput Stat Data Anal 53:4168-4177

Schnabel SK, Hurtado PX, Eilers PHC, Maliepaard C, Van Eeuwijk F (2012) Statistical tools for pre-processing phenotypic data. In progress

Sobotka F, Schnabel SK, Schulze Waltrup L (2012) Expectreg: expectile and quantile regression. $\mathrm{R}$ package, $0.35 \mathrm{edn}$

Stephens M (2013) A unified framework for association analysis with multiple related phenotypes. PLoS ONE 8:e65245

Struik PC, Ewing EE (1995) Crop physiology of potato (Solanum tuberosum): responses to photoperiod and temperature relevant to crop modelling

The Potato Genome Sequencing Consortium (2011) Genome sequence and analysis of the tuber crop potato. Nature 475:189-195

Turner AD, Ewing EE (1988) Effects of photoperiod, night temperature, and irradiance on flower production in the potato. Potato Res 31:257-268

van Eeuwijk FA, Bink MCAM, Chenu K, Chapman SC (2010) Detection and use of QTL for complex traits in multiple environments. Curr Opin Plant Biol 13:193-205

van Ooijen JW (2009) MapQTL 6. Software for the mapping of quantitative trait loci in experimental populations of diploid species. Kyazma BV, Wageningen

Verbeke G, Molenberghs G (2000) Linear mixed models for longitudinal data, 1st edn. Springer, New-York

VSNi (2012) GenStat reference manual, 15th edn. VSN International, Hemel Hempstead

Wolf JB, Frankino WA, Agrawal AF, Brodie E, Moore AJ (2001) Developmental interactions and the constituents of quantitative variation. Evolution 55:232-245

Wu R, Lin M (2006) Functional mapping-how to map and study the genetic architecture of dynamic complex traits. Nat Rev Genet 7:229-237

Wu R, Ma CX, Zhao W, Casella G (2003) Functional mapping for quantitative trait loci governing growth rates: a parametric model. Physiol Genom 14:241-249

Yin XY, Kropff MJ, McLaren G, Visperas RM (1995) A nonlinear model for crop development as a function of temperature. Agric Forest Meteorol 77:1-16 